This is a postprint version of the following published document:

Shafiq, Z., Cui, J., Pastor-Pérez, L., San Miguel, V., Gropeanu, R. A., Serrano, C. \& del Campo, A. (2012): Bioinspired Underwater Bonding and Debonding on Demand. Angewandte Chemie International Edition, 51 (18), pp. 4332-4335.

DOI: $\underline{10.1002 / \mathrm{adma} .201401290}$

(C) 2012 WILEY-VCH Verlag GmbH \& Co. KGaA, Weinheim 


\title{
Bioinspired Underwater Bonding and Debonding on Demand
}

\author{
Zahid Shafiq, Jiaxi Cui, Lourdes Pastor-Pérez, Verónica San Miguel, Radu A. Gropeanu, \\ Cristina Serrano, and Aránzazu del Campo*
}

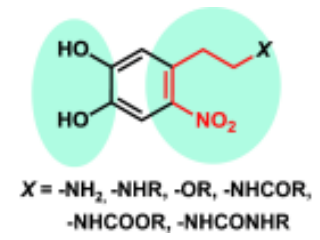

Mussel glue: Bioinspired underwater chemical bonding with the possibility of phototriggered debonding is reported. A four-arm star-poly(ethyleneglycol) endfunctionalized by nitrodopamine was synthesized. The nitrodopamine offered the reactivity of catechol and the chemistry of the photocleavable o-nitrophenyl ethyl group (see picture).
Adhesion under water, self-healing adhesives that stop catastrophic failure during use, and bonding/debonding on demand are three major topics in adhesion science and technology that are only partially resolved by using current gluing systems. Nature provides us with a fascinating example of underwater adhesion with self-healing properties in the mussel adhesive proteins. ${ }^{[1]}$ These proteins possess a high content of 3,4-dihydroxyphenylalanine (DOPA) residues. The basic $\mathrm{pH}$ and oxidant cations in sea water trigger catechol oxidation to quinone which further reacts to form a crosslinked polymer matrix. Robust binding to the rocks is achieved through chelation of the catechol functionality of DOPA with the inorganic oxides. Metal complexation between non-oxidized catechols and multivalent cations from sea water (i.e. $\mathrm{Fe}^{3+}$ ions) confers toughness to the adhesive structure because of the self-healing character of metal coordination complexes. ${ }^{[2]}$ In this way, a single organic functionality with a versatile chemical reactivity tuned by sea water triggers ( $\mathrm{pH}$, salt, ions) allows parallel improvement of interfacial and cohesive mechanical strength and durability in the mussel adhesive. Inspired by this system, several groups have used catechol-containing natural molecules for different applications: 1) to obtain stable surface anchors to metal oxide surfaces, ${ }^{[3]}$ 2) to generate multifunctional coatings onto almost any type of material, ${ }^{[4]}$ or 3 ) to incorporate them into polymer backbones as effective crosslinkers to obtain selfhealing networks ${ }^{[2 \mathrm{~d}]}$ or adhesive and biocompatible hydrogels $^{[5]}$ with unique clinical applications. ${ }^{[6]}$

Nitrocatechols (Figure 1A) are also natural occurring molecules (i.e. nitrodopamine and nitronorepinephrine are involved in NO metabolism and signalling pathways in neurons) with a similar crosslinking and complexation chemistry. The presence of the electron-withdrawing nitro group in the $p$-position lowers the $\mathrm{p} K$ of the catechol hydroxy groups $\left(\mathrm{p} K_{\mathrm{OH} 1} \approx 6.5\right.$ and $\left.\mathrm{p} K_{\mathrm{OH} 2} \approx 10\right)$, ${ }^{[7]}$ enhancing their acidity and hydrogen bond donor character and increasing stability against oxidation. Nitrocatechols are partially deprotonated at physiological $\mathrm{pH}$ and show higher affinity for coordination with metal oxides. ${ }^{[7]}$ But the most significant feature of the natural nitrocatechols and their derivatives has been overseen. The $o$-nitrophenyl ethyl moiety should be

[*] Dr. Z. Shafiq, ${ }^{[+]}$Dr. J. Cui ${ }^{[+]}$Dr. L. Pastor-Pérez, Dr. V. San Miguel, Dr. R. A. Gropeanu, Dr. C. Serrano, Dr. A. del Campo

Max-Planck-Institut für Polymerforschung

Ackermannweg 10, 55128 Mainz (Germany)

E-mail:delcampo@mpip-mainz.mpg.de photocleavable following the mechanism depicted in Figure $1 \mathrm{~A} \cdot{ }^{[8]}$ If this is the case, light exposure of a polymerized (through oxidative reactions or $\mathrm{M}^{n+}$ chelation) or a surfaceattached nitrocatechol derivative would trigger a molecular cleavage and, consequently, a phototriggered depolymerization reaction or surface detachment (Figure 1B). In other words, nitrocatechols represent the first example of bioinspired and self-healing underwater chemical bonding with the possibility of phototriggered debonding. Herein, we prove this hypothesis and show the potential of nitrocatecholamines to create biocompatible underwater self-curing, self-healing, surface-reactive, and photodegradable materials.

Nitrodopamine (ND) was readily synthesized by nitration of dopamine and was used to end-functionalize a four-arm star-poly(ethyleneglycol) (PEG-ND ${ }_{4}$ ) (see the Supporting Information for details). The presence of the ND end-groups was confirmed by ${ }^{1} \mathrm{H}$ NMR and UV spectroscopy (Figure SI1 in the Supporting Information). Covalently crosslinked gels were obtained under oxidative conditions $\left(\mathrm{NaIO}_{4}\right)$. The gelation time and crosslinking degree were adjusted by varying the concentration of the oxidant. Metal-mediated crosslinking of PEG-ND $\mathrm{N}_{4}$ was also obtained by adding $\mathrm{Fe}^{3+}$ cations to a concentrated aqueous solution of the polymer (molar ratio $\mathrm{FeCl}_{3}: \mathrm{ND}=1: 3$ ). Covalently and metal-coordinated homogeneous thin hydrogel films $(<1 \mu \mathrm{m})$ were obtained (see the Supporting Information for details).

The photoresponse of the PEG-ND ${ }_{4}$-crosslinked hydrogel films was studied using a quartz crystal microbalance with dissipation (QCM-D). The detected changes in the frequency $(\Delta f)$ and dissipation $(\Delta D)$ are indicators for changes in the mass and viscoelasticity of the hydrogel film supported by the quartz crystal. The use of a window-cell allowed in situ measurement of the changes of the hydrogel film during light exposure. Figure $2 \mathrm{~A}$ shows $\Delta f$ and $\Delta D$ of covalent and $\mathrm{Fe}^{3+}$ crosslinked films. Light exposure caused a continuous increase in $\Delta f$, indicating a mass decrease at the surface layer. This can be associated to the photocleavage of the $o$ nitrophenyl ethyl crosslinking units and the continuous removal of the hydrogel film from the surface. The lightdose-dependent removal of the hydrogel films from the substrate was further confirmed by a loss in absorbance detected by UV spectroscopy of hydrogel films supported onto quartz substrates after exposure and washing (Figure $3 \mathrm{~A}$ ). Covalently crosslinked films with increasing oxidant concentrations required higher exposure doses for cleavage (Figure 2B). Hydrogel films crosslinked with $20 \mathrm{~mm}$ oxidant concentration did not change upon exposure, indicating that too extensive crosslinking does not allow photoremoval of the gel at the employed irradiation doses. 

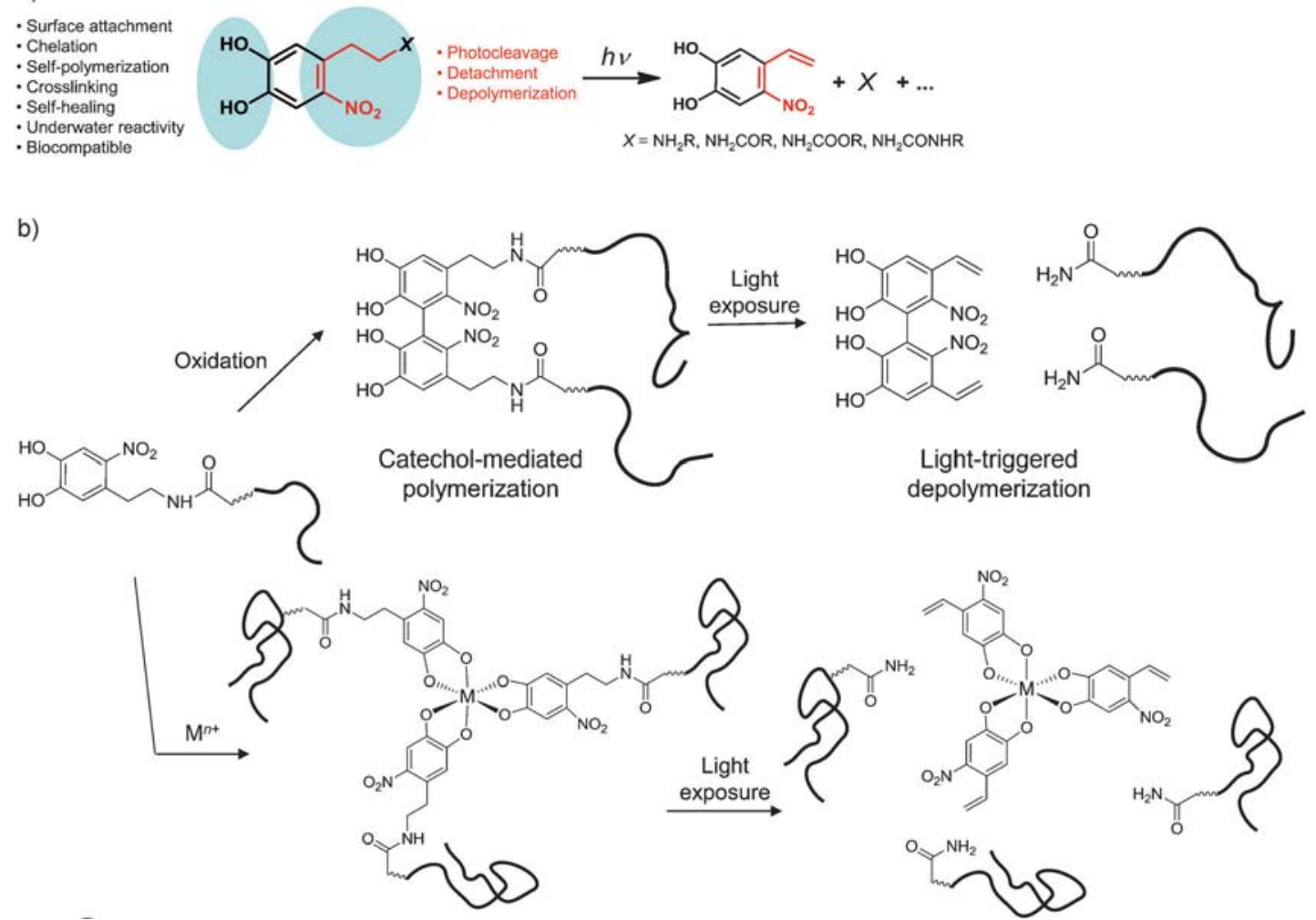
depolymerization

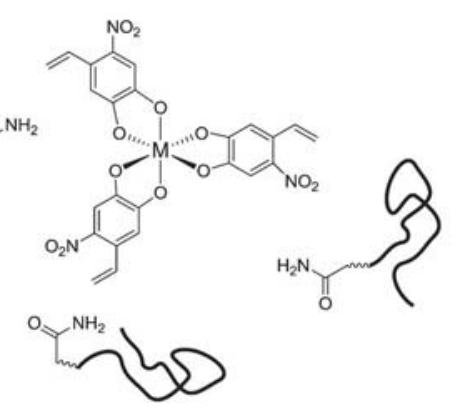

Figure 1. Structure of nitrodopamine derivatives and their photocleavage mechanisms. a) Photolytic reaction of the o-nitrophenyl ethyl moiety. See Figure SII in the Supporting Information for a more detailed reaction mechanism pathway. b) Different strategies used to trigger bonding (through oxidation of the catechol unit to quinone and further reaction, or through formation of metal complexes) and debonding upon exposure of nitrodopamine derivatives to light. Catechol reactions may lead to other polymerization products, but the photoreaction cleaves the linked chains in every case.

The photocleavage of the $\mathrm{Fe}^{3+}$-crosslinked gel was faster and more effective than that of the covalently crosslinked gels and, therefore, significantly lower exposure doses were required for complete removal of the film (Figure $2 \mathrm{~A}$ ). We do not expect differences in the photocleavage mechanism of covalently and $\mathrm{Fe}^{3+}$-crosslinked gels. However, the free catechols in the covalently crosslinked gel may well interfere with the reactive intermediates of the photolytic reaction and cause polymerization side-reactions that lower the overall depolymerization yield mediated by $o$-nitrophenyl ethyl photocleavage. UV and HPLC analysis of irradiated nitrodopamine in the presence and absence of catechol chelating units confirmed this hypothesis (data not shown). These sidereactions are less favored in the $\mathrm{Fe}^{3+}$-crosslinked gel at $\mathrm{pH}>$ 10 since the catechol groups are chelated forming the triscoordinated complex and, therefore, less prone to react with other groups.

The dissipation curves also reflected the changes in the hydrogel during exposure. In parallel to the mass loss, $\Delta D$ initially increased and afterwards decreased with increasing exposure dose in the covalently crosslinked films (5 and $10 \mathrm{~mm}$ oxidant). This behavior reflects that the viscoelasticity of the film increased at low photolytic conversion as a consequence of the small reduction in the crosslinking degree, and decreased afterwards while the film was gradually dissolved and removed from the crystal. The initial increase was not detected in the $\mathrm{Fe}^{3+}$-crosslinked gel, presumably because of the faster photocleavage process.

The light-triggered cleavage of the hydrogel was further visualized by irradiating thin hydrogel films through a mask with micrometric stripes. A clear pattern was observed after washing the film in water for a few seconds (Figure 2B). The gel was removed from the exposed regions and remained intact at the non-illuminated areas. These results firmly confirm the ability of ND to allow underwater bonding and light-triggered debonding of crosslinked materials.

We also tested the capability of nitrodopamine to form photocleavable and biocompatible coatings onto solid surfaces. Homogeneous and transparent nitrodopamine coatings were easily obtained by immersing quartz substrates into a $2 \mathrm{mg} \mathrm{mL}^{-1}$ solution of nitrodopamine in Tris- $\mathrm{HCl}$ buffer at $\mathrm{pH} 8.5$ for $24 \mathrm{~h}$. Upon light exposure and a short washing step, the absorbance significantly decreased (Figure 3B), evidencing the photocleavage of the $o$-nitrophenyl ethyl moiety in the structure and proving that phototriggered depolymerization had occurred. The photocleavage process leaves a residual monolayer of dopamine attached to the surface (the same holds for the hydrogel case). This is reflected in the residual absorbance after full exposure (green line in Figure 3B).

Cells were incubated onto the ND layers. Cells grew onto the nitrodopamine and retained their functionality, showing the biocompatibility of the coating (data not shown). 
At last we proved that nitrodopamine gels retain the self-healing property associated to the metal-coordinated catechol units. The $\mathrm{Fe}^{3+}$-crosslinked PEG-ND 4 gel was cut in two on the entire length of the gel piece. As depicted in Figure 2C, the crack was no longer visible after $3 \mathrm{~min}$. Moreover, distant pieces obtained by repetitive cutting of the gel, fused always together into a selfsupported single film (see Movie 1 in the Supporting Information). These results show that the nitro substitution does not affect the self-healing properties of metal-crosslinked catechol-derivatized gels.

In conclusion, natural nitrocatechols like ND are biocompatible crosslinkers able to form covalently and metal-crosslinked stable gels and coatings underwater that can be photodegraded upon light exposure. In this way, mussel-inspired materials functionalized with nitrocatechols retain all advantages of catechol bonding and add an additional important functional level: light-triggered debonding and degradation on demand. Taking account of 1) the relevance of DOPA-inspired materials as surgical glues and scaffolds for tissue regeneration $^{[9]}$ or biocompatible coatings, ${ }^{[4]}$ 2) the urgent need for reversible and degradable variants of the aforementioned materials (i.e. detachable hydrogel pads for skin regeneration, reversible glues for repetitive surgical operations, substrates for cell sheet engineering), and 3) the interest that dynamic materials enabling real-time and site-specific manipulation of their physicochemical properties using biologically friendly stimuli have for cell biology, drug delivery, or tissue regeneration, ${ }^{[10]}$ our results open new possibilities for the generation of photodegradable biomaterials with wide use in multiple cell and medical applications.
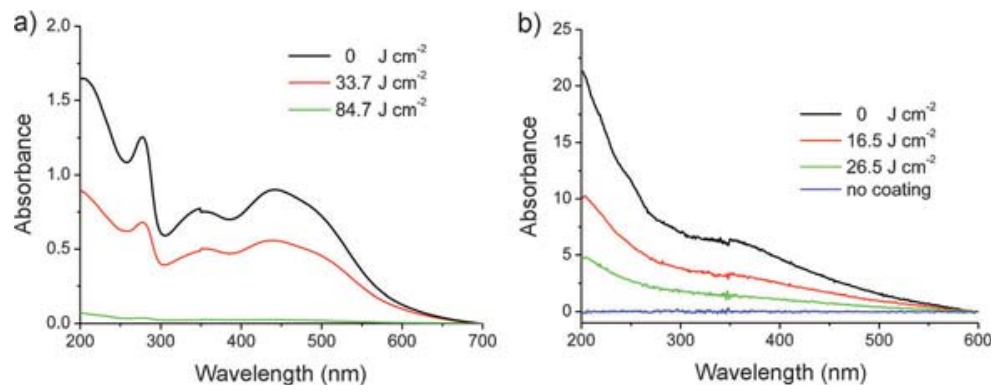
exposure with an increasing dose of light and a washing step. The decrease in absorbance is associated with the photocleavage of the o-nitrophenyl ethyl group and the release of the PEG chains attached to the chromophore from the film. A weak residual absorbance (almost not visible at this scale) remained after full irradiation, as expected from surface-bound ND molecules that cannot be cleaved. b) UV spectra of nitrodopamine coating deposited onto a quartz substrate upon irradiation at $360 \mathrm{~nm}$ with increasing doses and washing. The decrease in absorbance reflects the photo-depolymerization process and release of the ND coating from the substrate. The residual absorbance after full exposure (green line) corresponds to the surface-bound ND molecules.
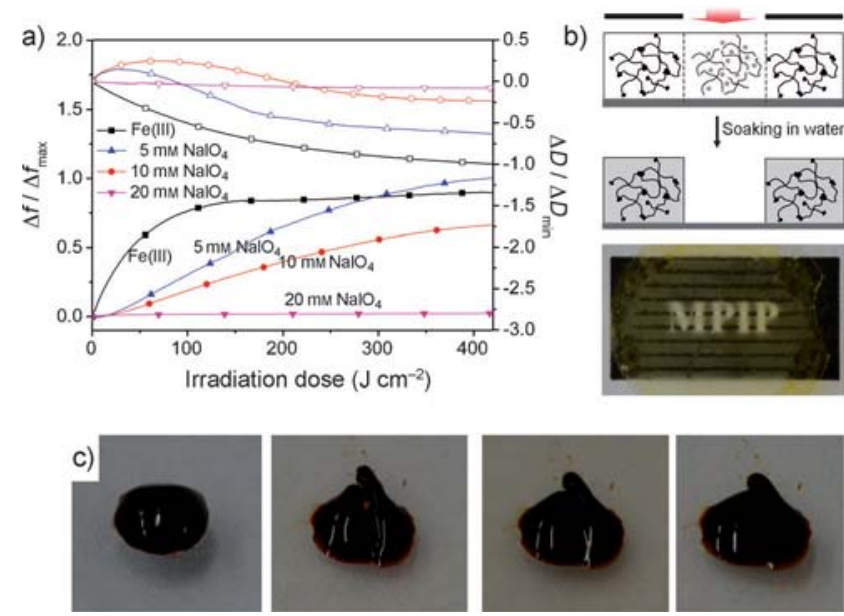

Figure 2. Photodegradation and self-healing properties of the PEG-ND gels. a) Normalized frequency (closed symbols) and dissipation (open symbols) changes of the third overtone of a QCM crystal coated with the PEG-ND ${ }_{4}$ film during exposure to light at $460 \mathrm{~nm}$. $\Delta f$ and $\Delta D$ were normalized by dividing through the maximum and minimum values, respectively, to take account of the differences in the film thickness between the different samples. The different curves correspond to films that were covalently crosslinked using different oxidant concentrations and to $\mathrm{Fe}^{3+}$-crosslinked films. b) Masked irradiation of a hydrogel film and microscopic image of hydrogel patterns obtained after masked exposure and development in water of a covalently crosslinked PEG-ND ${ }_{4}$ film (0.6 $\mu \mathrm{m}$ thickness). The bright stripes (200 $\mu \mathrm{m}$ width) correspond to exposed regions after washing, from where the gel was removed. c) Self-healing of PEG-ND ${ }_{4}-\mathrm{Fe}^{3+}$ gels after failure induced by cutting. The gel recovered its cohesiveness within minutes. 
[1] a) Q. Lin, D. Gourdon, C. J. Sun, N. Holten-Andersen, T. H. Anderson, J. H. Waite, J. N. Israelachvili, Proc. Natl. Acad. Sci. USA 2007, 104, 3782 -3786; b) J. H. Waite, Nat. Mater. 2008, 7, $8-9$.

[2] a) M. J. Sever, J. T. Weisser, J. Monahan, S. Srinivasan, J. J. Wilker, Angew. Chem. 2004, 116, 454-456; Angew. Chem. Int. Ed. 2004, 43, 448-450; b) M. J. Harrington, A. Masic, N. HoltenAndersen, J. H. Waite, P. Fratzl, Science 2010, 328, 216-220; c) A. Miserez, T. Schneberk, C. J. Sun, F. W. Zok, J. H. Waite, Science 2008, 319, 1816-1819; d) N. Holten-Andersen, D. M. Harrington, H. Birkedal, B. P. Lee, P. B. Messersmith, K. Y. C. Lee, J. H. Waite, Proc. Natl. Acad. Sci. USA 2011, 108, $2651-$ 2655.

[3] a) S. Saxer, C. Portmann, S. Tosatti, K. Gademann, S. Zurcher, M. Textor, Macromolecules 2010, 43, 1050-1060; b) M. Rodenstein, S. Zurcher, S. G. P. Tosatti, N. D. Spencer, Langmuir 2010, 26, 16211-16220.

[4] a) S. M. Kang, J. Rho, I. S. Choi, P. B. Messersmith, H. Lee, J. Am. Chem. Soc. 2009, 131, 13224-13225; b) H. Lee, Y. Lee, A. R. Statz, J. Rho, T. G. Park, P. B. Messersmith, Adv. Mater. 2008, 20, 1619-1623; c) H. Lee, S. M. Dellatore, W. M. Miller, P. B. Messersmith, Science 2007, 318, 426-430; d) H. Lee, J. Rho, P. B. Messersmith, Adv. Mater. 2009, 21, 431-434.

[5] a) M. Guvendiren, P. B. Messersmith, K. R. Shull, Biomacromolecules 2008, 9, 122-128; b) K. Huang, B. P. Lee, D. R. Ingram, P. B. Messersmith, Biomacromolecules 2002, 3, $397-$ 406; c) B. P. Lee, C. Y. Chao, F. N. Nunalee, E. Motan, K. R. Shull, P. B. Messersmith, Macromolecules 2006, 39, 1740-1748; d) B. P. Lee, J. L. Dalsin, P. B. Messersmith, Biomacromolecules 2002, 3, 1038-1047; e) G. Westwood, T. N. Horton, J. J. Wilker, Macromolecules 2007, 40, 3960-3964.

[6] P. B. Messersmith, C. E. Brubaker, H. Kissler, L. J. Wang, D. B. Kaufman, Biomaterials 2010, 31, 420-427.

[7] a) E. Reimhult, E. Amstad, A. U. Gehring, H. Fischer, V. V. Nagaiyanallur, G. Hahner, M. Textor, J. Phys. Chem. C 2011, 115, 683-691; b) S. Zurcher, B. Malisova, S. Tosatti, M. Textor, K. Gademann, Langmuir 2010, 26, 4018-4026; c) S. Zurcher, S. Saxer, C. Portmann, S. Tosatti, K. Gademann, M. Textor, Macromolecules 2010, 43, 1050-1060; d) E. Reimhult, E.

Amstad, T. Gillich, I. Bilecka, M. Textor, Nano Lett. 2009, 9 4042-4048; e) E. Reimhult, E. Amstad, S. Zurcher, A. Mashaghi, J. Y. Wong, M. Textor, Small 2009, 5, 1334-1342.

[8] a) D. Wöll, S. Walbert, K. P. Stengele, T. J. Albert, T. Richmond, J. Norton, M. Singer, R. D. Green, W. Pfleiderer, U. E. Steiner, Helv. Chim. Acta 2004, 87, 28-45; b) A. Specht, J. S. Thomann, K. Alarcon, W. Wittayanan, D. Ogden, T. Furuta, T. Kurakawa, M. Goeldner, ChemBioChem 2006, 7, 1690-1695.

[9] C. E. Brubaker, H. Kissler, L. J. Wang, D. B. Kaufman, P. B Messersmith, Biomaterials 2010, 31, 420-427.

[10] a) B. J. Adzima, Y. H. Tao, C. J. Kloxin, C. A. DeForest, K. S Anseth, C. N. Bowman, Nat. Chem. 2011, 3, 256-259; b) A. M. Kloxin, A. M. Kasko, C. N. Salinas, K. S. Anseth, Science 2009, $324,59-63$ 